\title{
Visual Design and Instructional Effectiveness
}

\author{
Seda Khadimally \\ School of Advanced Studies (SAS), University of Phoenix, Arizona, U.S.A.
}

\begin{abstract}
The paradigms of old media are replaced by new forms of creativity and delivery of content by those who strive to enhance innovative ways of communication and learning based on universally accepted guidelines. These ubiquitous rules of design are what scholars in the field of call as the principles of design. Particularly when instructional effectiveness of a new message is under close lens, instructional designers unanimously accept and implement these design principles in the process of planning and designing products for consumers and/or learners. With today's digital revolution and technological break-throughs, instructional designers need to keep seeking new forms of design, communication, and learning with various forms of instructional media and innovative, technology-rich design techniques. Given this educational milieu in an ever-growing digital world of learning and instruction, this paper will focus on eliciting some of these most innovative and outstanding instructional design (ID) products and evaluate their effectiveness in learning and teaching environments based on an array of ID principles generally accepted by the scholars of the field.
\end{abstract}

Keywords: visual design, instructional design (ID), communications, learning, teaching, English as a Second Language (ESL), ESL learning and teaching environments, adult learners

\section{Introduction}

According to Costello, Youngblood, and Youngblood (2012), "the principles of design can be thought of as the recipe for combining elements within a visual space" (p. 71). In this digital era where old theories, pedagogies, and methodologies of learning and instruction are superseded by new means and technologies of communication and learning, what calls for is an outside-the-box type of thinking that should shake these outdates models of normal science from its grassroots. As such, it is impossible to underestimate the role of today's digital revolution in education, especially with the surge of the Internet and the World Wide Web, in addition to other emerging multiple forms of media through which information is disseminated and shared in an online, digitized network and in an open system. With the digital revolution breaking through, therefore, instructional designers need to keep seeking new forms of design, communication, and learning with various forms of instructional media and innovative, technology-rich design techniques. Given this educational milieu in an ever-growing digital world of learning and instruction, this paper will focus on eliciting some of these most innovative and outstanding

Seda Khadimally, MEd, ALM (Harvard University Alumna), Doctor of Education (EdD) Candidate in Educational Leadership with Specialization in Educational Technology, School of Advanced Studies (SAS), University of Phoenix, Arizona, U.S.A. Instructor of Language \& Technology for Digital Natives Course and Scholarly Writing with the APA Manual Course at Bechtel International Center (I-Center), Stanford University, CA, U.S.A., Editor of the Forthcoming Publication, Technology-Assisted ESL Acquisition and Development for Nontraditional Learners. E-mail: sedakhadimally@email.phoenix.edu. 
instructional design (ID) products and evaluate their effectiveness in learning and teaching environments based on an array of ID principles generally accepted by the scholars of the field.

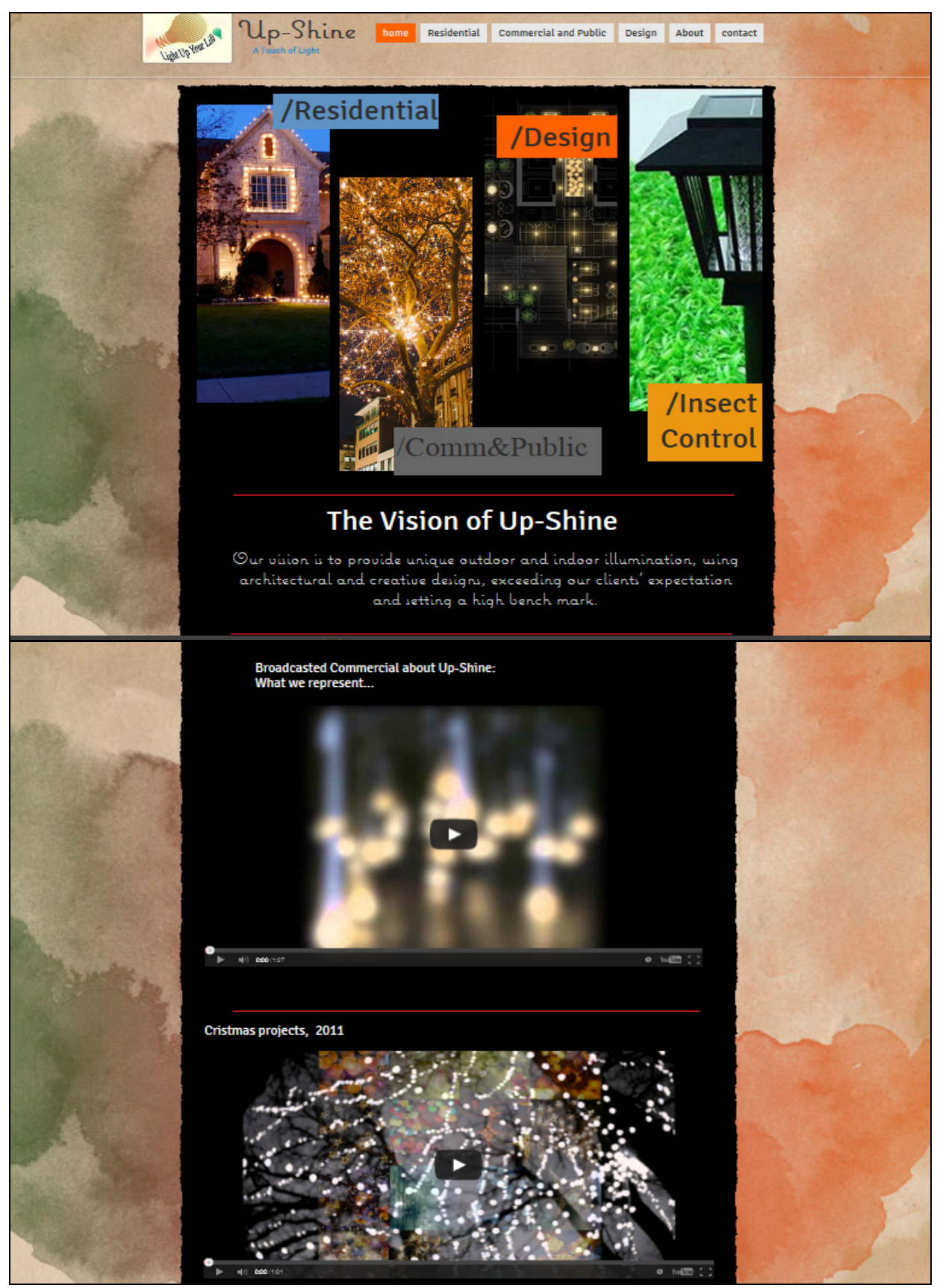

Figure 1. Example to a well-designed website: Up-Shine $L L C .^{1}$

\section{Evaluation of Visual Design Principles Applied}

Costello et al. (2012) described the principles of design as "formal rules and concepts for optimizing the arrangement and presentation of two-dimensional visual elements" (p. 71). Clark and Mayer (2011) focus on

\footnotetext{
${ }^{1}$ Source: http://ftkon0.wix.com/up-shine
} 
static illustrations when they recommend that instructional designers that they add graphics to their instruction. The examples to these static illustrations include "drawings, charts, graphs, maps, or photos, and dynamic graphics such as animation or video" (Clark \& Mayer, 2011, p. 70). The designers of this website seem to have applied these visual design principles in line with the authors' visual design-related guidelines.

\section{Unity}

According to Costello et al. (2012), unity is an essential ingredient of visual design, and they described it "as the perceptual glue that holds a design together and maintains a sense of visual harmony" (p. 71). Here, in this design product, all of the visual subcomponents of the entire website appear to work together in harmony and form some unity, for they render a unified meaning to the viewers, without distracting them. The holistic impression all of these separate components of the website give to the viewers contributes to the content as a whole, rather than separate parts of the message. Thus, they do not end up competing against one another or other elements in the design. In this way, the viewers can focus on the message. This website is solid medium of a message conveyed to viewers in a holistic manner and therefore, they are able to not only understand the message as it is, but also see the big picture, the story, or the narrative being told in a wonderfully dramatic manner. This brings to mind what McLuhan (1964) said, "the medium is the message" (as cited in Costello et al., 2012, p. 98).

\section{Emphasis}

With emphasis, instructional designers can maintain a visual focal point (Costello et al., 2012, p. 71). By taking emphasis design principle into account, instructional designers can determine what is pivotal or has weight as far as the content is concerned. This is a design principle "often used to communicate to the viewer the relative importance of visual objects or information in a design" (Costello et al., 2012, p. 71). Hence, the viewers do not lose their focus on, once again, the big picture depicted about the services of this lighting company targeting a niche clientele.

\section{Perceptual Forces}

These are the visual design elements that allow the viewers of this website grasp the content on a much deeper level. The website designers seem to have design this product in a way that appeals to the needs of the users in several ways such as in their residence, at work, on Christmas, everyday life, and the like. Due to the way they are visually affected by the content and interact with it on a rather personal level (e.g., decorating homes with Christmas lights, decoration of homes with indoors lighting, etc.), the viewers can fully engage, understand, and appreciate the value of the product presented and the services introduced.

\section{Instructional Effectiveness Evaluated}

Use of multimedia in communication and learning/teaching settings includes the "combination of text, graphics, video, audio, and animation through a digital channel or device" (Costello et al., 2012, p. 23). Use of visuals in this visual (i.e., website) enriches the content delivered, thereby paving the way for authenticity and ingenuity of the message intended to transmit to learners/users of the product. This facilitates learning, for research indicates that learners demonstrate higher interest in the message when they are given visuals rather than when they are presented text. All of the videos enriched with sounds and meanings of the messages amplified with written words particularly play an effective role in this learning environment. In support of this, Clark and 
Mayer (2011) posited that instructional designers can "use a combination of text and audio, as well as still and motion visuals to communicate [their] content" (p. 17). Because this website is a good example to a multimedia presentation of content, it additionally helps learners engage in the active learning process due to their ability to make connections between two types of representations: pictorial and verbal (Clark \& Mayer, 2011).

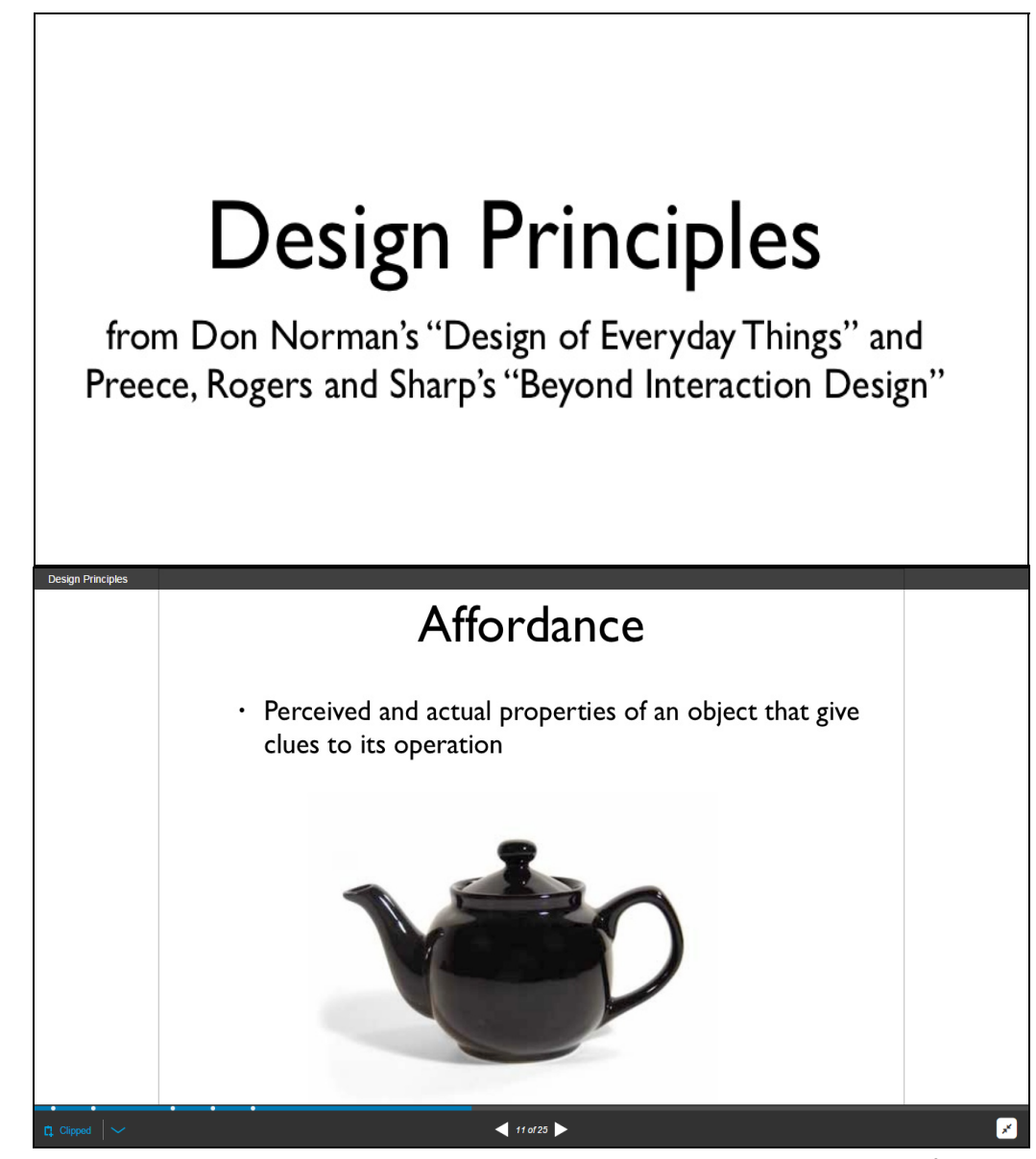

Figure 2. Example to a well-designed presentation (on SlideShare). ${ }^{2}$

\section{Evaluation of Norman's (2013) Six Visual Design Principles}

Norman (2013) provided designers with six visual design principles that can be used for everyday interaction. As demonstrated on the slides where viewers can see a teapot $-\mathrm{a}$ simple product used everyday - these design principles include visibility, feedback, affordance, mapping, constraint, and consistency. There seems to be a high level of aesthetics as each product is designed and all of the visual illustrations in this presentation successfully reflect each of these six design principles. According to Norman (2013), aesthetics comprises style and appearance that need to be reiterated in order to increase users' recognition of this product, as well as communicate a sense of membership to potential users and additionally need to set an emotional tone about that product. Norman (2013) said, "today, the products are beautiful, but for many of us, confusing. The fonts are pleasant to the eye, but difficult to read. The principle of 'discoverability' has been lost” (para. 3). When

\footnotetext{
${ }^{2}$ Source: http://www.slideshare.net/gelvan/design-principles
} 
one views each product visually depicted in this presentation, they are not only able to see the beauty of these products on an aesthetic level, but also understand their usability, especially where industrial design products are concerned.

\section{Are General Principles of Design Being Violated by Designers?}

Industrial products are becoming harder to use because of some of the general principles of design ignored by designers. This occurs because of the complexity of technologies today. However, the paradox is that things are actually getting to be designed based on a minimalist view. From his experience, Norman (2015) contended that industrial designers at the Apple Corporation have been following a rather minimalist design principle, lately. He commented that "Apple has gotten carried away by the slick, minimalist appearance of their products at the expense of ease of use, understandability, and the ability to do complex operations without ever looking at the manual" (para. 2).

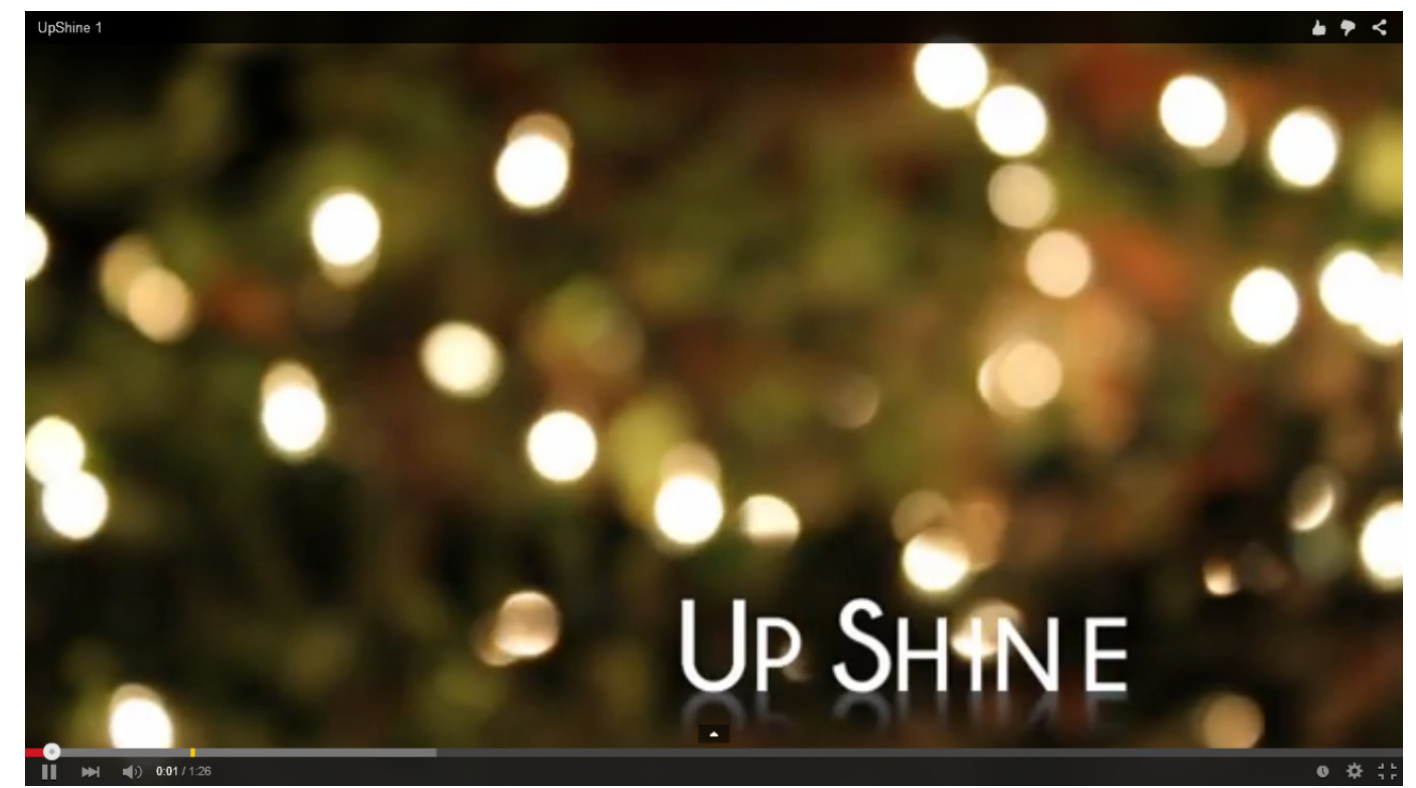

Figure 3. Good example to an End-of-the-Semester Video Project produced by students of Rice University's Advanced Business English Course facilitated and taught by Seda Khadimally. ${ }^{3}$

\section{Evaluation of Design Principles Applied With Additional Focus on CRAP and GESTALT}

All of the design principles addressed in this project were successfully applied by the students that designed this website. The students were asked by the instructor to invent a fictional company, prepare their company's logo, history, executive summary, business plans, organizational structure with a chart, a website with a clear page layout, one that potential clients can navigate and use, including their products and services, and even an infographic (see Figure 4 for evaluation on the infographic) which they seemed to have embedded in a quite humorous, yet informative manner.

\footnotetext{
${ }^{3}$ UpShine Website URL: https://www.youtube.com/watch?v=JHTCBsmkQNI UpShine Christmas Projects Video URL: http://ftkon0.wix.com/up-shine UpShine Unlimited Designs Video URL: http://ftkon0.wix.com/up-shine\#!design/ckra
} 


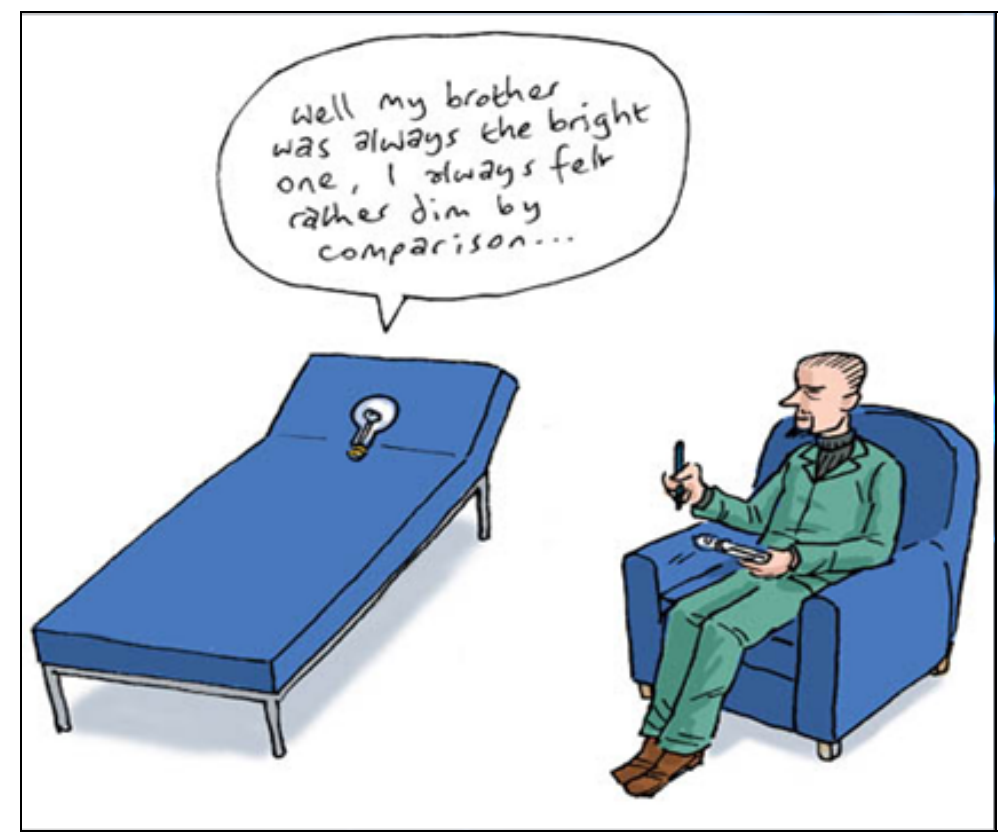

Figure 4. An infographic on UpShine LLC products. ${ }^{4}$

The UpShine LLC website comprises all of the generally accepted design principles that any industry designer or an instructional designer aspires to apply in their design works. Page Layout, Navigation, Usability, and other design considerations such as the saturation of colors and various levels of brightness, or value as Costello et al. (2012) put it, as well as Robin Williams' four popular visual design principles that involve contrast, repetition, alignment, and proximity (CRAP) (Kimball, 2013, p. 24) were all implemented effectively. Clearly, these novice website designers had a plan, a roadmap through which they focused on achieving unity and alignment among all of the design elements they used. Costello et al. (2012) argued that "a carefully hatched plan provides direction, a road map for helping you get to your intended destination. Without it, you will have no idea where you are going or how to get there" (p. 71).

It is also apparent that these novice designers concentrated on not only the message itself, but on how they were to convey this message; that is, the medium. McLuhan (1964) stated that medium is the message. The student designers did not merely create this visual design to create a WOW factor in the eyes of the viewers. In order to keep the attention of their viewers, they clearly focused on transmitting meaningful content on each page they designed and each piece of information they entered pertinent to their invented company, UpShine LLC. It is undoubtedly that these young designers did not want three things: (1) create a visual that would create an eye candy effect (Costello et al., 2012); (2) lose their viewers' or prospective customers' interest as they delved into the content; and (3) let the visual design dominate their message or vice versa. The last guideline they followed brings to mind what McLuhan (1964) said about the alignment between the medium and the message. McLuhan (1964) stressed that "if the form of the medium dominates the content, the message can be adversely affected" (as cited in Costello et al., 2012, p. 98).

\footnotetext{
${ }^{4}$ Source: This infographic was retrieved from the UpShine LLC PPT Presentation, which students of the Advanced Business English Course at Rice University Intensive English Program (IEP) were required to create as part of their end-of-the-semester, culminating start-up business project.
} 


\section{Infographic Evaluated Based on Visual Design Principles}

Each of the six Gestalt laws that include "alignment, proximity, grouping, closure, continuation, and figure/ground" (Kimball, 2012, p. 10) can be easily observed in this infographic related to the UpShine lighting and decoration company. The designers seem to have apply the Gestalt design principles in order to accentuate users' understading of the products and services of the company, which have to do with light. The operatuonal element, lightbulb, is highly instrumental in viewers' grasp of the focal point, which adds emphasis to the message of the designers. Unity is additionally sustained with the Gestalt design laws applied. This leads up to a holistic view on a visual level. In terms of meanings conveyed in this visual, it is possible to see a word play with the use of contrasting words: bright and dim, which particularly brings to mind the interplay between words and visuals for a successful, unified visual design. Clark and Mayer (2011) argued that "providing relevant graphics with text is a proven method of fostering deeper cognitive processing in learners. In short, learning is facilitated when the graphics and text work together to communicate the instructional message" (p. 74).

\section{Use of Sense of Humor}

According to Fresco (2010), an online programs instructor (New York on the Clock on PBS), having a sense of humor is a critical trait of instruction. The fact that the designers of this infographic used the humor element with users of their website - or their future clients - is a very important method to establish good, close relationships with them with compassion and ease. Use of both etiquette and appropriate humor with this visual can not only empower the interactions between the designers and their users, but can also be good detreminant of the level of receptivity of their potential customers. What Moore and Kearsley (2012) stated about adding elements of emotional support and social contact between learners and instructors can be adapted in this context to that between website users, infographic viewers, or future clients and the website designers themselves.

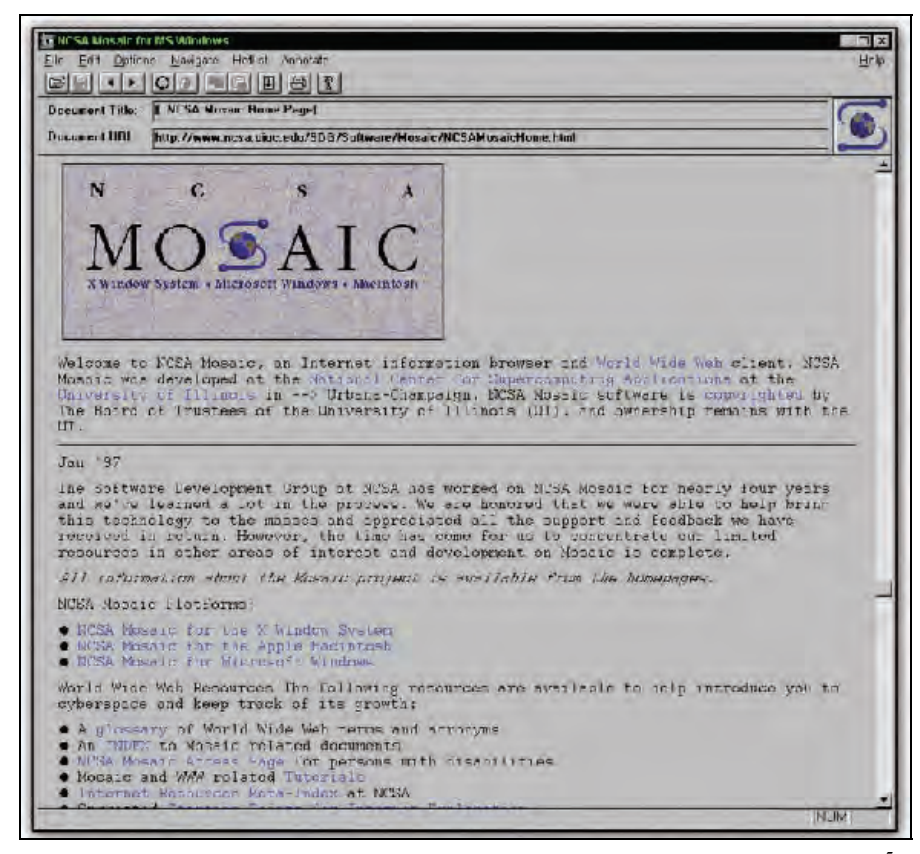

Figure 5. Example to a poorly-designed static instructional graphic. ${ }^{5}$

\footnotetext{
${ }^{5}$ Source: Costello et al., 2012
} 


\section{Visual Design Principles and Instructional Effectiveness of the Content Evaluated}

With the advent of the Internet back in 1980, and especially with the introduction of the World Wide Web on a massive, global scale, there has been a remarkable transformation of computer-based distance education in pretty much all educational systems around the world. Moore and Kearsley (2012) emphasized that "the first Web browser, called Mosaic, appeared in 1993, and it was this software that gave educators a powerful new way of opening access to learning at a distance" (p. 40). The authors' statement clearly demonstrates the powerful role of the fifth-generation, web-based, computer technology in the dissemination of knowledge across diverse, distant locations. However, because of the highly ill design choices made in the design of this static graphic, it is not easy to clearly decipher the elements on the interface, such as colors, contrasts, unity, alignment, emphasis, and others. There are no color contrasts and all colors used are desaturated, which, once again, hinders clear sight. Psychologists link the use of colors to brain-based learning, for it has long been used as an ideal technique for memorization (Costello et al. 2012).

Other poor design-related choices in this visual involve issues with perspective, proximity, continuity, and brightness, all of which are absent. First of all, this instructional graphic lacks unity, because none of the elements used allows for visual harmony as far as the users are concerned. Second, maintaining a visual focal point looks unfeasible because of the aforementioned subcomponents that were misplaced or poorly placed across the screen. This obstructs viewers or users to focus. Thus, they miss the weight and importance, or the emphasis, of the instructions on the screen. Third, the users are unable to navigate through the page, because they are unable to use the elements embedded into the design and they cannot interact with the content designers intended to transmit. For all these reasons, the entire design impedes clear visibility, navigation, and usability. It is especially impossible to find in this graphic Williams' visual design principles, which are contrast, repetition, alignment, and proximity (CRAP) (Kimball, 2013, p. 24). As such, it is not viable to discuss an effective instructional content here.

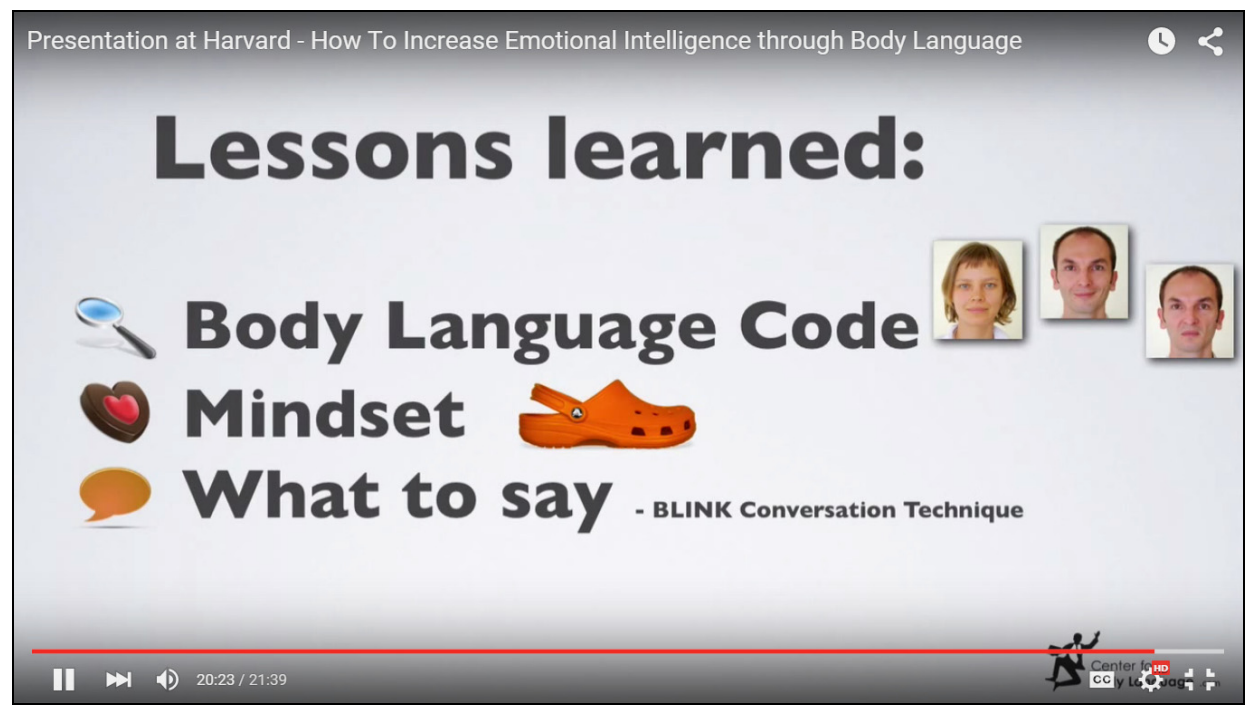

Figure 6. Example to a well-designed instructional video (How to Increase Emotional Intelligence Through Body Language). ${ }^{6}$

\footnotetext{
${ }^{6}$ Source: https://www.youtube.com/watch?v=uHjCh2SwZas
} 


\section{Design Principles and Instructional Effectiveness Evaluated}

Given the role of computer technology in the evolution of visual design, it is noteworthy that the manner in which today's instructional designers utilize these tools immensely contribute to learners' constructing meanings from within the designed and delivered content. This instructional video creates such a positive learning environment wherein learners find the subject authentic and engaging. With the speakers directing questions to viewers in the streaming video, learners can fully engage in the content. This not only facilitates learners' understanding, but also leads instructional designers to continue to use design elements that align with three major design principles (i.e., unity, emphasis, and perceptual forces). This video can also help leverage learners' critical thinking skills and optimize their ability to put themselves in others' shoes.

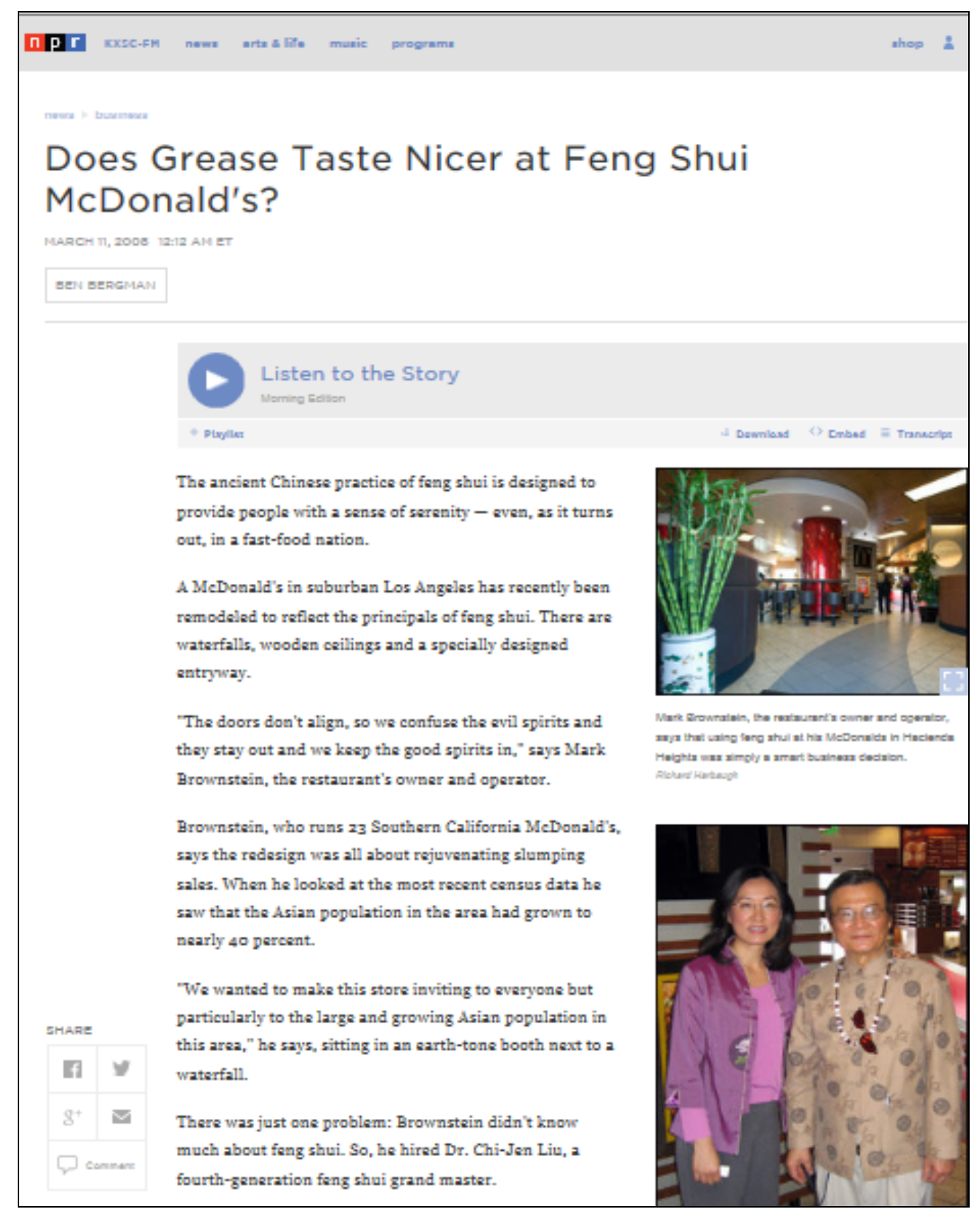

Figure 7. Example to an audio presentation (Podcast $\rightarrow$ Does Grease Taste Nicer at Feng Shui McDonald's?). 


\section{Instructional Design Principles and Instructional Effectiveness Evaluated}

This podcast, which is an audio presentation, provides the reader with a good example of how four design principles when designing audio instruction: (1) Selection of the narrative format; (2) fleeting nature of spoken words; (3) environmental soundscape; and (4) difference between hearing and listening. Ferrington (1994a) contended that "the goal of good audio design is to effectively engage the listener in active and attentive listening" (p. 67, as cited in Carter, 2012, p. 55). Both the weakness and the strength of the audio medium, according to Arnheim (1986), is a "blind medium that relies on the elements of sound and silence to communicate information or emotional content to the listener" (as cited in Carter, 2012, p. 55).

Audio created good design principles play a crucial role in effective learning and instruction, for they positively contribute to the learning processes of especially learners who have lost their sight. In other words, this podcast can certainly facilitate blind students' listening skills. However, the target learner group(s) the author of this paper focuses on for the purposes of this paper is not the individuals with this disability. On the other hand, because she specializes in teaching yet another non-traditional learner population, limited English Proficient (LEP) and/or linguistically and culturally diverse students, her aim by including in this paper this specific audio presentation - among many other multimedia designs - is to demonstrate that her LEP learners can and do significantly enhance their listening skills in the English language, which is not their native tongue to listen to and speak with. For instance, the third audio instruction design principle requires a thorough understanding of the Environmental Soundscape (e.g., attending to foreground or background sounds in the podcast). According to Ferrington (1994b), "active listening involves the ability to discriminate between sounds within a multi-pattern context" (as cited in Carter, 2012, p. 56). Regarding this (instructional) design principle, Carter (2012) posited that this "multi-pattern context (soundscape) has three layers through which the listener will isolate, identify, integrate, inspect, and then interpret spoken words to seek meaning in the sounds" (p. 56). This podcast undoubtedly paves the way for such a learning environment where the listeners can navigate through all of these three layers pertinent to the environmental soundscape incorporated.

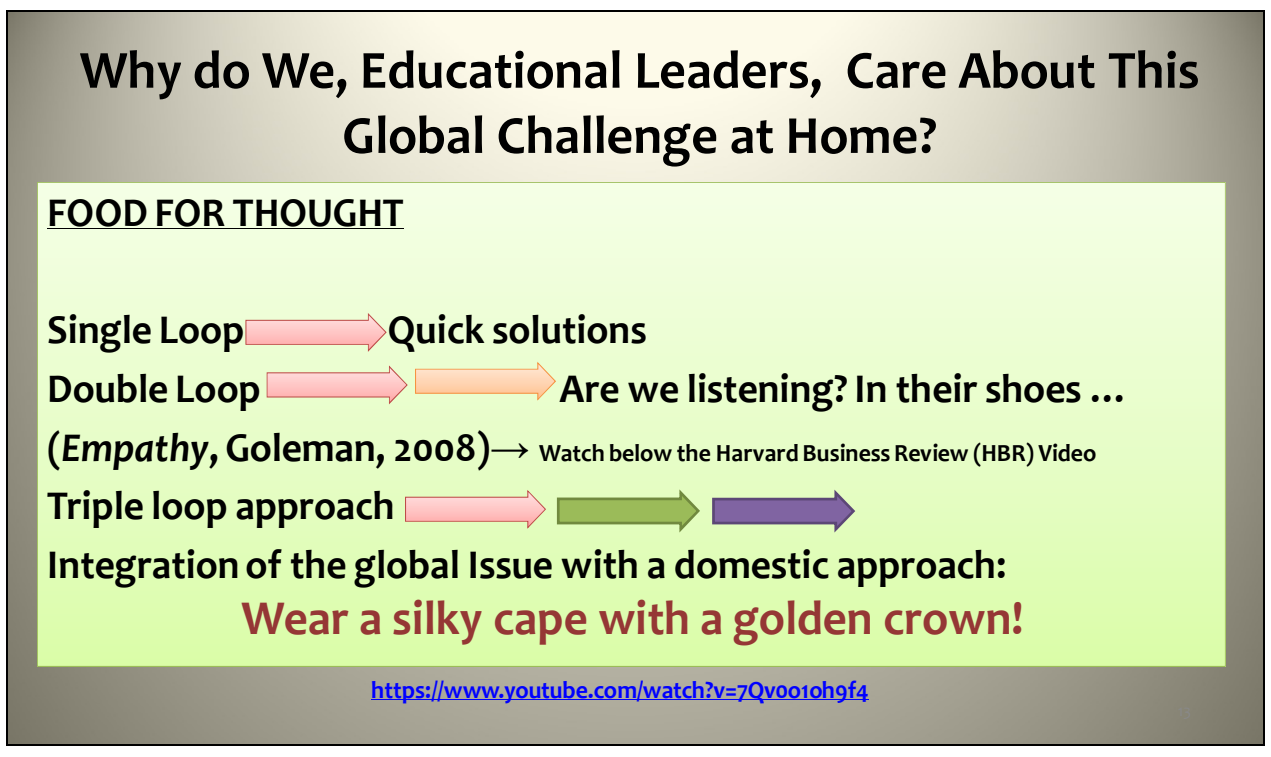




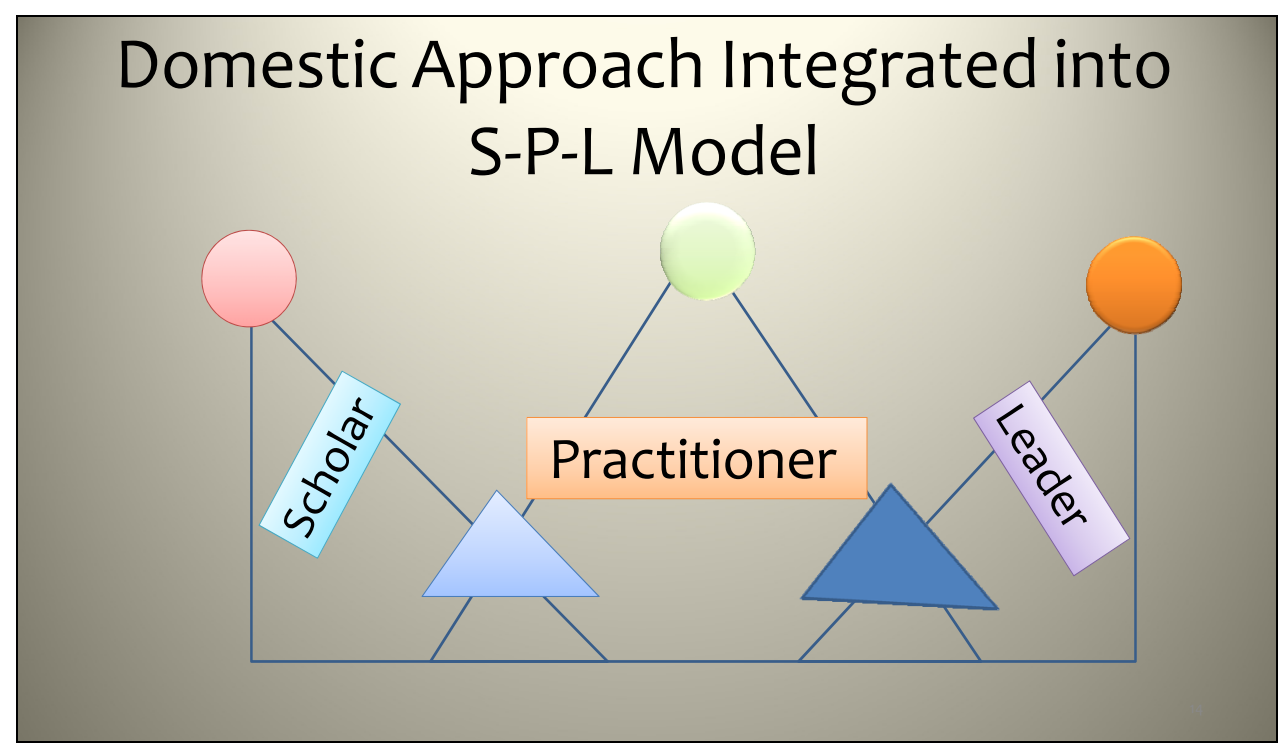

Figure 8. Two complementary examples to effective static instructional graphic.

\section{Eight Visual Design Elements Evaluated}

Costello et al. (2012) proposed eight visual design elements through which to maintain effective visual communication and learning, which are "space, dot, line, shape, form, texture, pattern, and color" (p. 101). Colors on these slides can be evaluated here in terms of their hue, saturation, and brightness (Costello et al., 2012). The hue, which is "the color shade of an object as a single point on the color spectrum" (Costello et al., 2012, p. 113) and brightness of all of the colors on the crown (i.e., SPL model) appear to have been successfully applied. Although all of the colors are desaturated (e.g., pink and orange) instead of saturated, they appear to maintain brightness.

Overall, all of these eight visual design components seem to have been accomplished by the designers of these (Microsoft Office) PowerPoint presentation slides. Especially the message conveyed at the bottom of the first slide ("Wear a silky cape with a golden crown!") completely foreshadows and unequivocally aligns with what is to follow on the next slide (i.e., a crown drawn by the members of this design), which is the University of Phoenix (UoPX) Scholar, Practitioner, and Leader (SPL) model. Hence, it is possible for the viewers or learners to see the unity of the content in a holistic manner. The viewers do not get distracted by redundant elements (e.g., colors, additional drawing, charts, pictorials, or other graphs) included in the background or they do not find the message elusive or intricate for themselves. In fact, the message is clearly and concisely transmitted. Also, the designers seem to have put an emphasis on what is being conveyed on the first slide, and successfully transmit the message here as the focal point of the subject (Costello et al., 2012) in a predominantly visual format, rather than solely through words.

These two slides are excellent representations of how an instructional designer should follow the principles of unity, emphasis, and perceptual forces. They also successfully take into account other design-related considerations including slide (page) layout, viewers' navigation through each element on these slides, and whether they can easily use the information presented on them (i.e., usability). On a final note, in terms of the effectiveness of the instructional message provided on these two visuals, it is important to add that, "providing 
relevant graphics with text is a proven method of fostering deeper cognitive processing in learners. In short, learning is facilitated when the graphics and text work together to communicate the instructional message" (Clark \& Mayer, 2011, p. 74). Drawing on this information, this instructional message on the SPL model is communicated to the viewers and/or learners highly effectively.

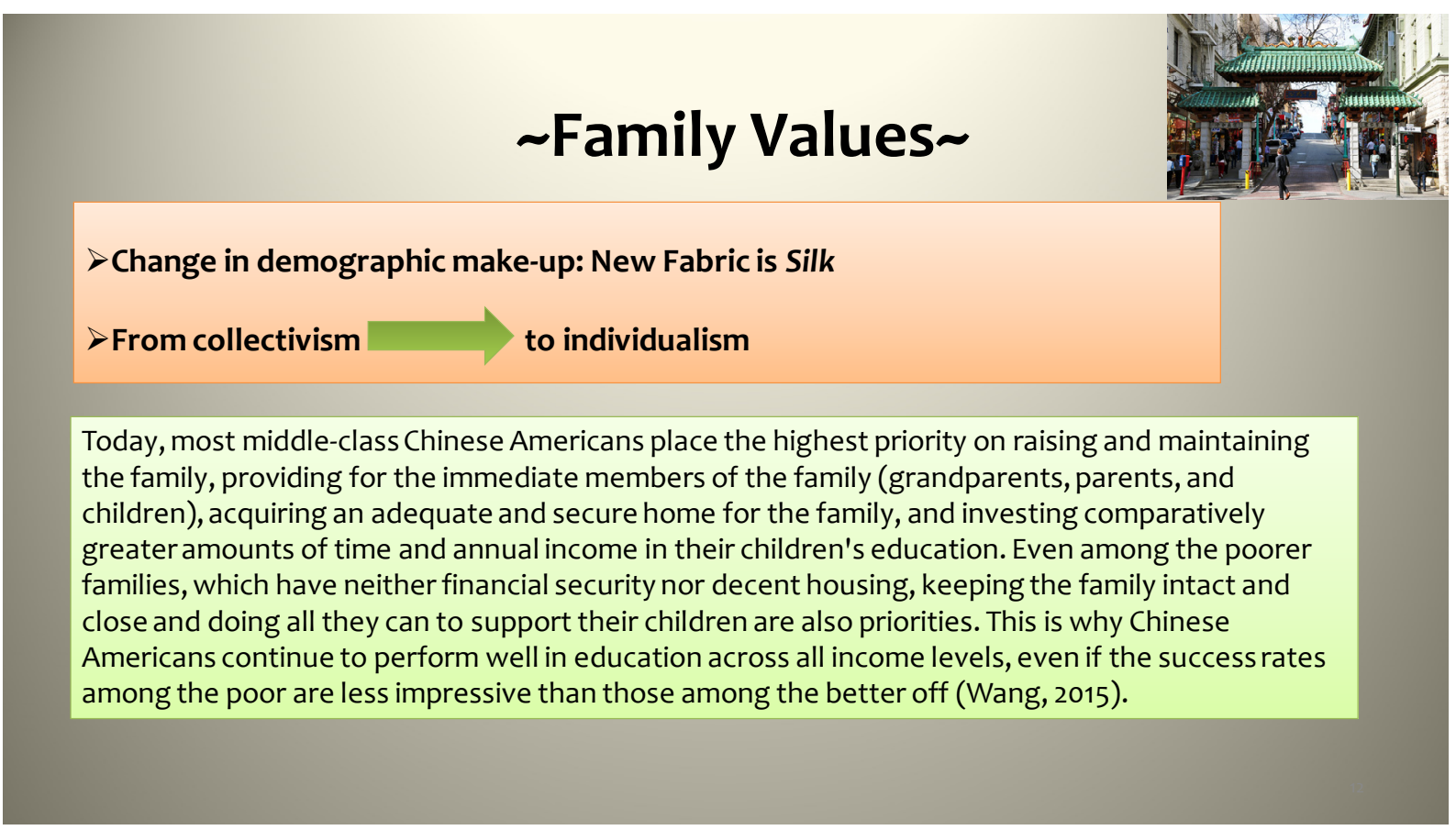

Figure 9. Example to a poorly-designed static instructional visual.

\section{Visual Design Principles Evaluated: Decorative Graphic in Use}

In this static instructional visual, a graphic (i.e., a picture of the traditional China Town) was incorporated into the visual for decorative purposes. Clark and Mayer (2011) called this poor design choice as a "decorative graphic" (p. 71) that may sometimes be redundant, superfluous, or even disconnected from the content. In this visual, the decorative graphic causes designers to digress from the major topic, by distracting the viewers. The decorative graphic additionally creates a medium wherein the message actually becomes elusive and/or even complicated for the viewers to understand.

\section{Instructional Effectiveness}

When effective ID principles are combined with use of appropriate visuals or technologies in learning and teaching environment, they allow for multiple modes of representation. They suggest alternative learning methods simultaneously, such as auditory and visual. However, if designers make poor choices on adding decorative graphics into their visual designs, they face challenges of confusing viewers or complicating what needs to be a simple message. For a holistic understanding and unity of the design product, they thus need to eliminate such poor design-related decisions. After all, including these forms of graphics can actually lead to messages that are relevant and thus can encourage learners engage in the active learning process. Clark and Mayer (2011) introduced multimedia principles in e-learning and psychological reasons behind using words and 
pictures together with various media forms for data representation in ID. It is noteworthy that the authors focus on multimedia (design-related) principles supported with engaged, active learning and cognitive processing. Regarding this, Mayer (2003) contended that "adding relevant graphics to words can be a powerful way to help learners engage in active learning" (as cited in Clark \& Mayer, 2011, p. 79).

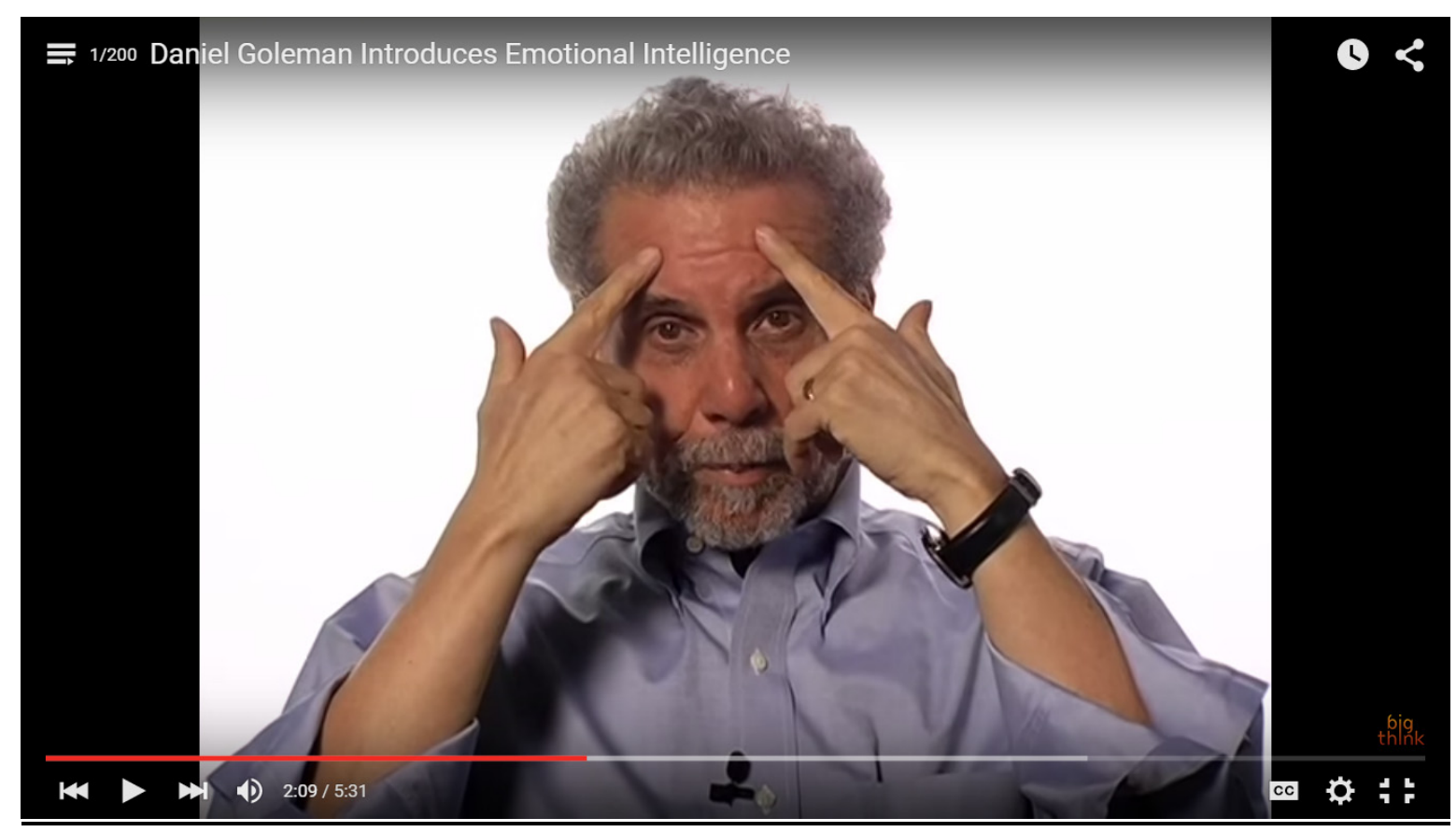

Figure 10. Example to a well-designed instructional video. $^{7}$

\section{Evaluation of Instructional Effectiveness and Perceptual Forces in This Visual Design}

Based on the role of the cognitive processing view in visual design, it is important to remember that blending visual information with verbal information can help designers achieve effective learning on the part of the product users or learners in an educational environment. Clark and Mayer (2011) explained this approach to learning as one in which "providing relevant graphics with text is a proven method of fostering deeper cognitive processing in learners. In short, learning is facilitated when the graphics and text work together to communicate the instructional message" (p. 74). If so, the manner in which the designer of this video on Daniel Goleman's Emotional Intelligence theory transmits the message is successful.

The designer of this video seems to have carefully and wisely selected the medium in which he aimed at putting the message across. After all, all of the spoken words, written words, visuals pertinent to the topic, as well as the body language Goleman himself uses, such as his hand gestures, leaning forward to give the impression to the viewer that they are somehow connected on a deeper level, and other instructional techniques positively contribute to the viewers' understanding of and appreciation for the content. These instructional methods also highly align with one of the major visual design principles that a majority of the visual design scholars agree upon: perceptual forces. Viewers can understand the message on a more profound level, because they connect with the speaker and relate to him both verbally and visually, which further facilitates their psychological learning

${ }^{7}$ See https://www.youtube.com/watch?v=Y7m9eNoB3NU\&list=PLJ3965bD3NKLvju47meyi0d7uun8pNZ-N 
processes. In this context, it is thus critical to keep in mind that in the design process, multimedia presentations can help learners engage in the active learning process due to their ability to make connections between two types of representations: pictorial and verbal (Clark \& Mayer, 2011).

\section{Conclusion}

In his critically acclaimed work, The Structure of Scientific Revolutions, Kuhn (1996) must have foreseen what was to incarnate in the neo-millenial educational milieu when he adamantly proposed new paradigms of thinking, learning, and communication. He provided that old methodologies and pedagogies of normal science are dysfunctional and that there needs to be a shift from the old cannons of science and education to the new. With this knowledge in mind, contemporary instructional designers use state-of-the-art technologies to create authentic and innovative products that lead users or learners to engage in higher cognitive processes.

Clark and Mayer (2011) stressed that today's communication and learning platforms give way to the emergence of cutting edge educational technologies that can be "delivered in many forms - such as printed words, spoken words, illustrations, photos, graphs, animation, video, and narration” (p. 79). Today, thanks to these multiple resources for consumption and learning, users and learners of the digital world think critically, solve problems, retain information, engage, and most importantly, construct their own meanings. One expectation with the evaluations in this paper was for readers to broaden their horizon regarding this new paradigm shift in technology in education, especially about new, multiple modes of instructional media incorporated into the generally accepted visual design principles. It was also the hope that readers could gain a new found appreciation for this digital media revolution and the new paradigm shift that manifests itself in everyday use of simple things that surround all individuals (Norman, 2013). As Costello et al. (2012) put it, "a paradigm shift of epic proportions has occurred and things will never be the same" (p. 15).

\section{References}

Arnheim, R. (1986). Radio: An art of sound. New York: Da Capo Press.

Carter, C. W. (2012). Instructional audio guidelines: Four design principles to consider for every instructional audio design effort. TechTrends, 56(6), 54-58.

Clark, R. C., \& Mayer, R. E. (2011). E-learning and the science of instruction (3rd ed.). Hoboken, NJ: Pfeiffer.

Costello, V., Youngblood, S.A., \& Youngblood, N. E. (2012). Multimedia foundations: Core concepts for digital design. Burlington, MA: Taylor \& Francis.

Ferrington, G. (1994a). Audio design: Creating multi-sensory images for the mind. Journal of Visual Literacy, 14(1), 61-67.

Ferrington, G. (1994b). Keep your ear-lids open. Journal of Visual Literacy, 14(2), 51-61. Retrieved from http://jcp.proscenia.net/publications/articles_mlr/ferrington/earlids.html

Kimball, M. A. (2013). Visual design principles: An empirical study of design lore. Journal of Technical Writing \& Communication, $43(1), 3-41$.

Kuhn, T. (1996). The structure of scientific revolutions. Chicago, IL: The University of Chicago Press.

Mayer, R. E. (2003). Learning and instruction. Upper Saddle River, NJ: Merrill Prentice Hall.

Moore, M. G., \& Kearsley, G. (2012). Distance education: A systems view of online learning (3rd ed.). CA: Cengage Learning. New York on the Clock (PBS). (2010). Distance adult educator [video]. Retrieved from http://video.pbs.org/video/1570666495/

Norman, D. A. (2013). Design of everyday things: Revised and expanded. New York: Basic Books. London: MIT Press (UK Edition).

Norman, D. A. (2015). Apple's products are getting harder to use because they ignore principles of design. Retrieved from $\mathrm{http}: / /$ www.jnd.org/dn.mss/apples_products_are.html 